\title{
Extrapolation and interpolation by time-scaling in systems with diffusion-controlled kinetics and first-order reaction rates
}

\author{
S. E. A. T. M. VAN DER ZEE, W. H. VAN RIEMSDIJK \& J. J. M. VAN \\ GRINSVEN
}

Department of Soil Science and Plant Nutrition, Wageningen Agricultural University, P.O. Box 8005, NL 6700 EC Wageningen, Netherlands

Received 22 June 1988; accepted 3 December 1988

\begin{abstract}
The interpolation or extrapolation in time of physico-chemical processes from experimental data is often difficult. A theoretically derived time-scaling procedure using an exposure variable of the concentration integrated in time, with at most one adjustable parameter was applied successfully to three systems taken from metallurgical, agricultural and environmental engineering. This scaling rule is potentially useful as a research or management tool for systems that are too complex for mechanistic modelling.
\end{abstract}

Keywords: reaction kinetics, mass transfer, shrinking score, exposure, phosphate, aluminium, acid deposition

\section{Introduction}

In chemical, metallurgical, and mining engineering as well as the earth sciences, kinetic processes are often of considerable importance. Kinetics play a role in e.g. ore processing, fuel combustion and oil shale or coal gasification, catalyst regeneration, mineral weathering, and fertilizer nutrient immobilization. Different rate-determining processes may be involved in such reactions. These processes are: diffusion, heat transfer, or reaction kinetics. Because the degree of conversion is a function of time, the dimension of the system of interest affects its effectivity. Thus in chemical reactor plants, the fluid velocity must be regulated for optimal results, and for optimal fertilization the moment and method of fertilizer application are under constraints. Reaction kinetics may depend on a large number of parameters and variables. Therefore, system optimization usually requires the evaluation of the effects of important variables on the reaction kinetics and performance of the system. Even if such an assessment is done only over a limited range of values of the dominant variables the effort may be large. To cut down on this effort, the system is often described mathematically. Mathematical expressions are used to fit the data, 
and for interpolation and extrapolation to conditions not evaluated experimentally. Using empirical models for this purpose may cause large uncertainties. The problem is simpler if the processes involved are understood physically. Then the mathematical formulation may be based on a realistic physico-chemical model. A particularly useful approach is to use non-dimensional mathematical equations, or dimensionless numbers such as the Reynolds and Schmidt numbers (Levenspiel, 1972). The use of such dimensionless quantities is based on scaling rules and was discussed extensively by Bird et al. (1960).

In this paper, we present a time-scaling method. The theoretical background is well documented in chemical engineering literature of solid-fluid and solid-gas interactions for relatively simple systems. Processes in such systems are dominated by diffusional transfer or first-order reaction kinetics. We show that such time scaling may be feasible, even if the mechanisms are incompletely understood, for complicated reaction systems. The method is illustrated with examples from different fields of interest.

\section{Background of scaling}

The overall kinetics of conversion of a solid phase may be controlled by e.g. the diffusion rate of one of the reactants, the heat transfer rate if heat is produced or consumed, and the concentration. Other factors that may be of importance are e.g. solid characteristics such as specific surface area, solid phase purity, etc. In the case of an isothermal, non-catalytic reaction, the conversion rate (the rate of change of the reacted amount of solid phase relative to the total amount present initially) may be fitted to mathematical functions with at least one parameter. Often, several empirically fitted rate equations may seem equally appropriate. Moreover, equations with more than one parameter may yield similar results for different values of the parameter. Therefore, little physical relevance can be attributed to apparent rate constants obtained in this way. For example the square root of time behaviour of unsteady linear diffusion can be described equally well with an empirical expression for the reaction rate (Skopp, 1986). Experimental errors and the similarities in the description of different physico-chemical processes complicate the discrimination between different mathematical descriptions. However, such similarities may be of use if mechanistic understanding of the overall conversion process is small. This is illustrated by considering the conversion of solid phase particles.

The conversion rate is controlled by three resistances, acting in series. The mechanisms causing these resistances are:

1) diffusive mass transfer through a stagnant fluid film surrounding a particle,

2) intraparticle diffusion through a layer of converted solid,

3) first-order chemical reaction rate.

A model taking these three mechanisms into account is the unreacted shrinking core (USC) model (Levenspiel, 1972; Wen, 1968). In the USC model the unconverted solid cannot be penetrated by the gaseous or liquid reactant. By reaction at the surface of the unconverted solid, a reaction product layer forms that grows at the expense of the unreacted and shrinking core. In the different homogeneous 
conversion model $(\mathrm{HC})$, reactant diffuses into the unreacted solid and the reaction occurs in the entire particle. In general, the USC model is physically most realistic (Levenspiel, 1972; Wen, 1968). Because the discrimination of the two models is seldomly possible on the basis of data (Wen, 1968) the approach is illustrated with the USC model.

The exact solution of the mathematical equations describing the USC model has received much attention in applied mathematics literature. Usually the problem to solve the moving boundary problem of the USC model is to find a suitable transformation of variables (Ockendon \& Hodgkins, 1975, Davis \& Hill, 1982). The mathematics are considerably simpler if we assume pseudo steady state. This assumption was challenged (Davis \& Hill, 1982; Bischoff, 1963) but is acceptable for gas-solid reactions or the reactions between dilute electrolytes and a solid (Wen, 1968). Early solutions of the USC model assumed that the overall conversion rate is controlled by one of the resistances (Yagi \& Kunii, 1954). Later extensions included mixed control (Lu, 1963), a reversible reaction (Lu \& Bitsianes, 1966), and swelling or shrinking of the particle (Shen \& Smith, 1965). A pseudo steady state solution is commonly presented, by considering the fractional conversion $(X)$, as a function of dimensionless time. Dimensionless time is obtained by division of real time $(t)$ with the time $(\tau)$ required for a complete conversion.

The fractional conversion $(X)$ equals the amount of material that has reacted divided by the amount of material that initially was present (at $t=0$ ). Thus for spherical geometry with initial particle radius of $R_{0}$, and a radius at time $t$ of the unconverted core equal to $R_{\mathrm{c}}$ we have:

$$
X=1-R_{\mathrm{c}}^{3} / R_{\mathrm{o}}^{3}
$$

We assume that the three resistances act in series. Then, if one of the resistances is rate-controlling, we can derive expressions giving the time $(t)$-conversion $(X)$ relation. For an ensemble of equally sized and shaped particles the solutions are given by:

$$
t=\tau_{i, j} P_{i, j}(X) \quad i, j=1,2,3
$$

in which the index $i$ denotes the rate-controlling process or resistance and the index $j$ denotes the particle geometry.

Equation 2 implies that $\tau_{i, j}$, which is a function of different parameters, and $P_{i, j}(X)$, which is a function of $X$, differ for different $i$ and $j$. In Table 1 the functions $P_{i, j}(X)$ are given for different cases. We see that if the data conform to a linear $t(X)$ relationship the conversion process may be described assuming each of the three geometries, or with two rate-limiting steps for planar geometry, alike. The expressions relating $\tau_{i, j}$ to solid and solution parameters are, however, different for these cases. Hence, the parameters lumped in $\tau_{i, j}$ can not be determined uniquely from data giving $t(X)$ for such cases.

An important point is that the expressions for $\tau_{i, j}$, when compared, in each case show that $\tau_{i, j}$ is inversely proportional to $c$, the concentration of the reactant in the 
Table 1. Relationships $P_{i, j}(X)$ between fractional conversion $(X)$ and dimensionless time $t / \tau_{i, j}$ with $i$ the rate controlling step, and $j$ identifying particle geometry.

\begin{tabular}{lllll}
\hline$j$ & Geometry & $\begin{array}{l}i=1 \\
\text { Film diffusion }\end{array}$ & $\begin{array}{l}i=2 \\
\text { Product layer diffusion }\end{array}$ & $\begin{array}{l}i=3 \\
\text { Reaction rate }\end{array}$ \\
& & & \\
1 & plate & $P_{11}=X$ & $P_{21}=X^{2}$ & $P_{31}=X$ \\
2 & cylinder & $P_{12}=X$ & $P_{22}=X+(1-X) \ln (1-X)$ & $P_{32}=1-(1-X)^{0.5}$ \\
3 & Sphere & $P_{13}=X$ & $P_{23}=1-3(1-X)^{2 / 3}+2(1-X)$ & $P_{33}=1-(1-X)^{1 / 3}$ \\
\hline
\end{tabular}

bulk of the solution. This implies that for mixed conversion control, when the resistances are of comparable importance, instead of one rate-controlling resistance, we may write:

$$
t=\sum_{i} \tau_{i, j} P_{i, j}(X)
$$

where only one geometry is concerned $(j=$ constant $)$. For the total conversion time we then have:

$$
\tau_{\text {total }}=\sum_{i} \tau_{i, j} \quad j \text { constant }
$$

Furthermore, if we substitute:

$$
\tau_{i, j}=\tau_{i, j}^{*} / c
$$

into Equation 3 we obtain:

$$
t=\frac{1}{c} \sum_{i} \tau_{i, j}^{*} P_{i, j}(X)
$$

where $\tau^{*}$ is independent of $c$. The expression found by rearrangement of Equation 6 is a main result of this analysis and may be given as:

$$
c t=\sum_{i} \tau_{i, j}^{*} P_{i, j}(X)
$$

or:

$$
c t=F(X)
$$

where $F(X)$ is a function of $X$.

By inversion we may also obtain an explicit expression of $X$, i.e.:

$$
X=F^{-1}(c t)
$$

This result may be formulated in words as: 'there is a unique, though not necessarily known, functional relationship between the conversion of an ensemble of particles 
with the same size and shape and the product of concentration and time'. Because Equation 8 holds for different geometries and rate-controlling resistances, also for ensembles of differently shaped particles a unique relationship holds.

That a unique relationship between $X$ and $c t$ can also be expected for ensembles with particle size distributions was shown numerically (Bartlett, 1971; Braun et al., 1974; Szekely \& Propster, 1975) and analytically (van der Zee, 1988). Sofar the analysis leading to the concentration-scaled conversion time expression was based on the assumption of a constant bulk concentration in the gas or liquid phase. However, if this bulk concentration varies slowly in time and if the pseudo steady state approximation is valid, scaling of time is still possible. In that case an integral of $c$ over $t$ should be used instead of the variable $c t$. This integral, that resembles the exposure variable I commonly used in toxicology (Brunekreeff, 1985), may be defined as

$$
I=\gamma \int\left(c-c_{e}\right) d t
$$

where $\gamma$ is a parameter that may be taken equal to unity and that is used to make $I$ dimensionless. Instead of concentration we use the concentration difference $(c-$ $\left.c_{e}\right)$, because in many cases it is realistic to identify a lower (no-effect) level of $c,(c=$ $c_{e}$ ), below which a reaction does not occur. Such a lower bound might be governed by e.g. solubility of the reaction product. For first-order chemical reaction rate control, the scaling holds also for fast variations in time of $c$.

With the extended USC model presented so far, the process of conversion is described well for an ensemble of well-defined particle shapes and sizes, if a large number of model parameters such as reaction stoichiometry, diffusion coefficients and chemical rate constants are known. If, however, these constants are not well known or if solid phase impurity, solid phase mixtures, etc. complicate the system, then the USC model as such is of limited use. In that case, deterministic modelling becomes practically impossible due to the large number of parameters, that may differ for different solid phase constituents, and that are hard to assess experimentally. Unfortunately, many natural systems studied in e.g. the earth and environmental sciences are usually very complex. In such systems, where mechanistic kinetic models are not applicable, the time-scaling method using the exposure integral will still yield good results because the unique relation between $X$ and $I$ is still valid. So, even for systems that are incompletely understood, interpolation in the exposure domain covered experimentally is possible. An important consequence of the use of the exposure variable is that a long reaction at a small concentration is equivalent to a short reaction at a large concentration, if $I$ is the same for both cases. Thus, extrapolation to long reaction times (and small concentrations) may be done by assessing $X(I)$ for short-duration experiments at high concentrations.

The theory developed in this paper was based on a simplified description originating from gas-solid reaction models. To test whether it is applicable also to reaction between fluids and porous media, the scaling model was applied to three rather different examples discussed in the next section. 


\section{Materials and methods}

\section{Application 1: Galvanic conversion of chalcopyrite}

This conversion process was studied by Hiskey \& Wadsworth (1974) whose experimental data were used. Two samples of chalcopyrite $\left(\mathrm{CuFeS}_{2}\right)$ were used with small impurities of quartz and pyrite. High-purity copper shot $(100 \times 150 \mathrm{mesh})$ and appropriate portions of chalcopyrite were mixed and transferred to the reaction vessel containing $\mathrm{H}_{2} \mathrm{SO}_{4}$-solutions of designated concentrations $(0.05 ; 0.1 ; 0.5 ; 1.0 ;$ or 2.0 $\mathrm{mol} \mathrm{l}^{-1}$ at a temperature of $90^{\circ} \mathrm{C}$. The extent of the reaction was monitored by taking $5 \mathrm{ml}$ solution samples at desired time intervals and analysis of the iron concentration with a Perking-Elmer model 305 atomic absorption spectrophotometer.

\section{Application 2: Phosphate reaction with acid, sandy soil}

Topsoil (0-20 cm depth) was taken from a field in Barneveld. Forty samples per hectare were thoroughly mixed, air-dried and sieved $(2 \mathrm{~mm})$. Some properties of this soil were described by van Riemsdijk \& van der Linden (1984). Phosphate sorption was measured according to van Riemsdijk \& van der Linden (1984) on $30 \mathrm{~g}$ of soil mixed with $30 \mathrm{~g}$ of quartz sand, and a $10 \mathrm{~mol} \mathrm{KCl} \mathrm{m}{ }^{-3}$ background electrolyte solution. Experiments were performed at constant $\mathrm{P}$-concentrations in the range of 0.05 to $6 \mathrm{~mol} \mathrm{P} \mathrm{m}^{-3}$, in duplicate. An additional experiment was done for a P-concentration starting at about $4 \mathrm{~mol} \mathrm{~m}^{-3}$ and decreasing to $0.05 \mathrm{~mol} \mathrm{~m}^{-3}$ at the termination of the experiment. The value of $I$ was found from the measured concentrations at different times as:

$$
I \approx \gamma \sum_{i}\left(\frac{c_{i-1}+c_{i}}{2}-c_{e}\right)\left(t_{i}-t_{i-1}\right) \quad i \geqslant 1
$$

where $c_{0}$ is the concentration after addition of $P$ at $t_{0}=0, c_{i}$ is the measured concentration at $t_{i}, c_{e}$ is a fitting constant that was found to be equal to $0.1 \mathrm{~mol} \mathrm{~m}^{-3}$, time $t$ is in minutes and $\gamma$ equals $1\left(\mathrm{~mol}^{-3} \mathrm{~m}^{3} \mathrm{~min}^{-1}\right)$. Sorption $(F)$ was fitted to a third-order polynomial of $\ln (I): F=a+b \ln (I)+c[\ln (I)]^{2}+d[\ln (I)]^{3}$.

\section{Application 3: Proton consumption by mineral weathering}

The soil samples were derived from an acid sandy soil (Umbric Dystrochrept), i.e., the C-horizon at 70-80 cm depth (van Grinsven, 1988). Chemical analysis and column percolation experiments at constant $\mathrm{pH}$ using a new technique were described by van Grinsven (1988). After 3 to 10 days of percolation at different adjusted $\mathrm{pH}$ values, experiments were continued in batch with the same material and at the same pH's. Each time the proton concentration had decreased by about $200 \mathrm{mmol}$ $\mathrm{l}^{-1}$ (in a $15 \mathrm{ml}$ solution), a $100 \mathrm{ml}$ of the solution were sampled and replenished (once in 1-3 weeks). These experiments had a duration of 90 days. The batch reaction vessels were not shaken. The constant $\mathrm{pH}$ values used were $3,3.5$, and 3.9 for column and batch alike. The amounts of released $\mathrm{Si}$ and $\mathrm{Al}$, as well as base cations (van Grinsven, 1988b) were measured. 


\section{Results}

\section{Applications of time scaling}

In this section we show three applications of the scaling method which differ in the degree in which the reaction mechanisms are known in detail. The first example is the galvanic conversion of chalcopyrite $\left(\mathrm{CuFeS}_{2}\right)$ by metallic copper. This metallurgical process was described by the USC model (Bartlett, 1971, 1973). The over-all reaction is given by:

$$
\mathrm{CuFeS}{ }_{2}+\mathrm{Cu}^{0}+2 \mathrm{H}^{+}=\mathrm{Cu}_{2} \mathrm{~S}+\mathrm{Fe}^{2+}+\mathrm{H}_{2} \mathrm{~S}
$$

The over-all reaction rate depends on the rate of proton diffusion to the unconverted chalcopyrite surface and the proton consumption rate at this surface. For relatively narrow ranges of the particle sizes of chalcopyrite and metallic copper, the conversion was studied experimentally (at $90^{\circ} \mathrm{C}$ ) (Hiskey \& Wadsworth, 1974). The protons were supplied by adding $\mathrm{H}_{2} \mathrm{SO}_{4}$ at different concentrations. In Figure 1a some results are given for different, constant concentrations. In accordance with our scaling method, a single line (Fig. 1b) is found when the fractional conversion is given as a function of $c t$.

The next example is the reaction of orthophosphate with soil, which is of interest from the scope of $P$ fertilizer efficiency and for environmental reasons. Due to the reaction in soils containing little phosphorus, fertilizer-P becomes less available as the time after the moment of fertilizer application increases (Rahman, 1982; van der Zee \& van Riemsdijk, 1988). Hence, the application effectivity decreases also. In soil receiving large quantities of $\mathrm{P}$, leaching of $\mathrm{P}$ may eventually cause surface water eutrophication (Beek, 1979; Enfield et al., 1981). In non-calcareous soil, phosphate reacts predominantly with iron and aluminum oxides (Beek, 1979).

The overall reaction that consists of adsorption in combination to a diffusion-type process (Barrow, 1983; van Riemsdijk et al., 1984; van der Zee \& van Riemsdijk, 1986) can be described as a function of exposure $(I)$ and measured according to van Riemsdijk \& van der Linden (1984). In Figure 2a the amount of phosphate reacted as a function of $\ln (t)$ is shown for four different constant concentrations. The logarithmic time scale was taken for convenience to compress the horizontal axis as measurement time varied from $1 \mathrm{~min}$ to $40 \mathrm{~h}$. A total of ten different concentrations were considered experimentally but only four are shown in Figure $2 a$. In Figure $2 b$ we show the scaled data for all ten concentrations. Also shown is a best-fitting thirdorder polynomial of $\ln (I)$. Considering the complexity of phosphate-soil interaction, the scaling result can be considered excellent.

In the same way we could describe the reaction of phosphate with soil for times of approximately one year (van der Zee \& van Riemsdijk, 1988), so that the results could be used to dimension waste disposal sites. The use of this approach to evaluate laboratory experiments is illustrated in Figure 3. Using the same experimental set up (van Riemsdijk \& van der Linden, 1984), a phosphate-containing solution was percolated through a column containing the same soil as used in Figure 2 . The 

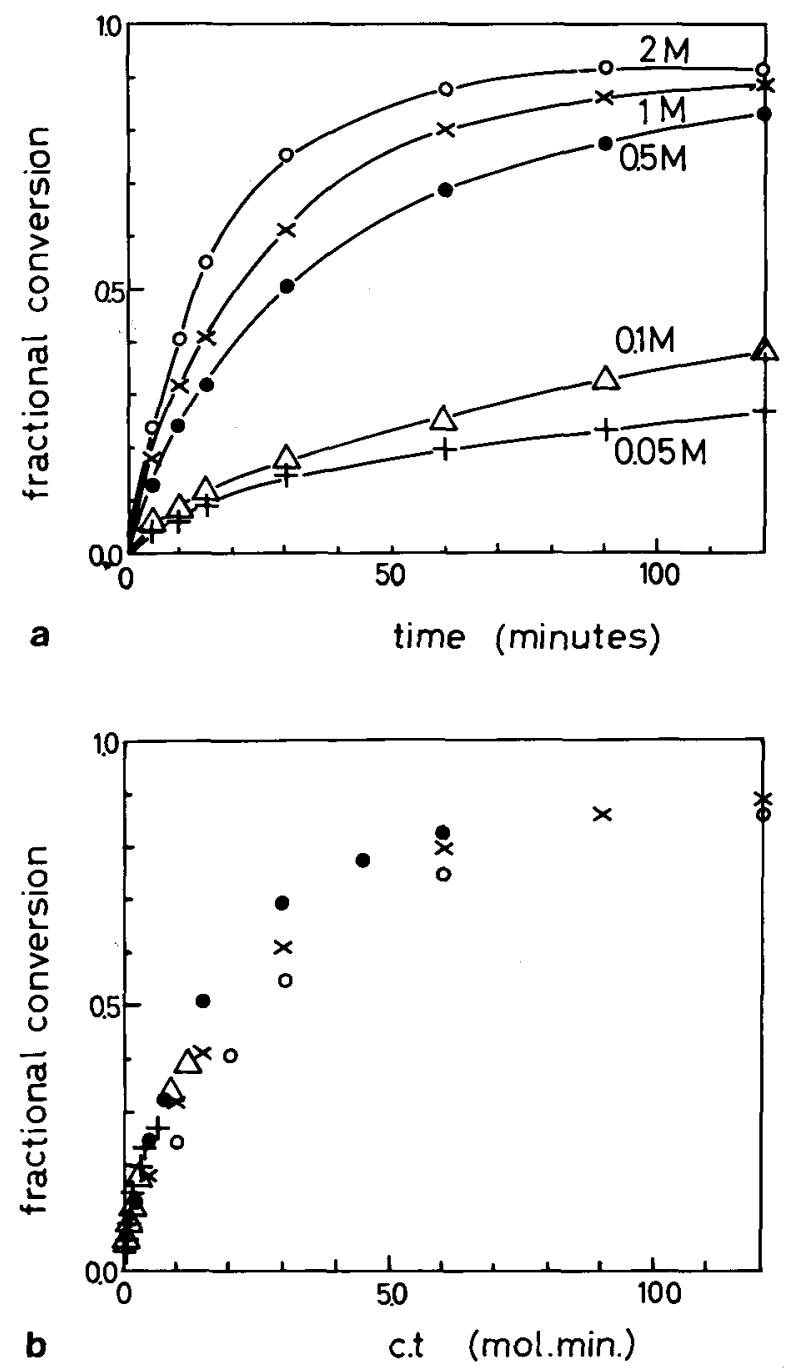

Fig. 1. Fractional conversion of chalcopyrite $\left(\mathrm{CuFeS}_{2}\right)$ as a function of time (1a; after Hiskey \& Wadswalk, 1974), and as a function of $c t(1 \mathrm{~b})$. Concentration (c) of $\mathrm{H}_{2} \mathrm{SO}_{4}$ as indicated in 1a.

concentration was not kept constant and therefore decreases by the reaction. The change in concentration as a function of time was predicted using the polynomial function of Figure $2 b$, and proven to be in excellent agreement with the measured concentrations. These results illustrate the applicability of the scaling procedure at variable concentrations.

A third example is taken from in the field of soil acidification. Atmospheric deposition of $\mathrm{SO}_{\mathrm{x}}, \mathrm{NO}_{\mathrm{x}}$ and $\mathrm{NH}_{3}$ on the soil causes soil acidification which affects the 

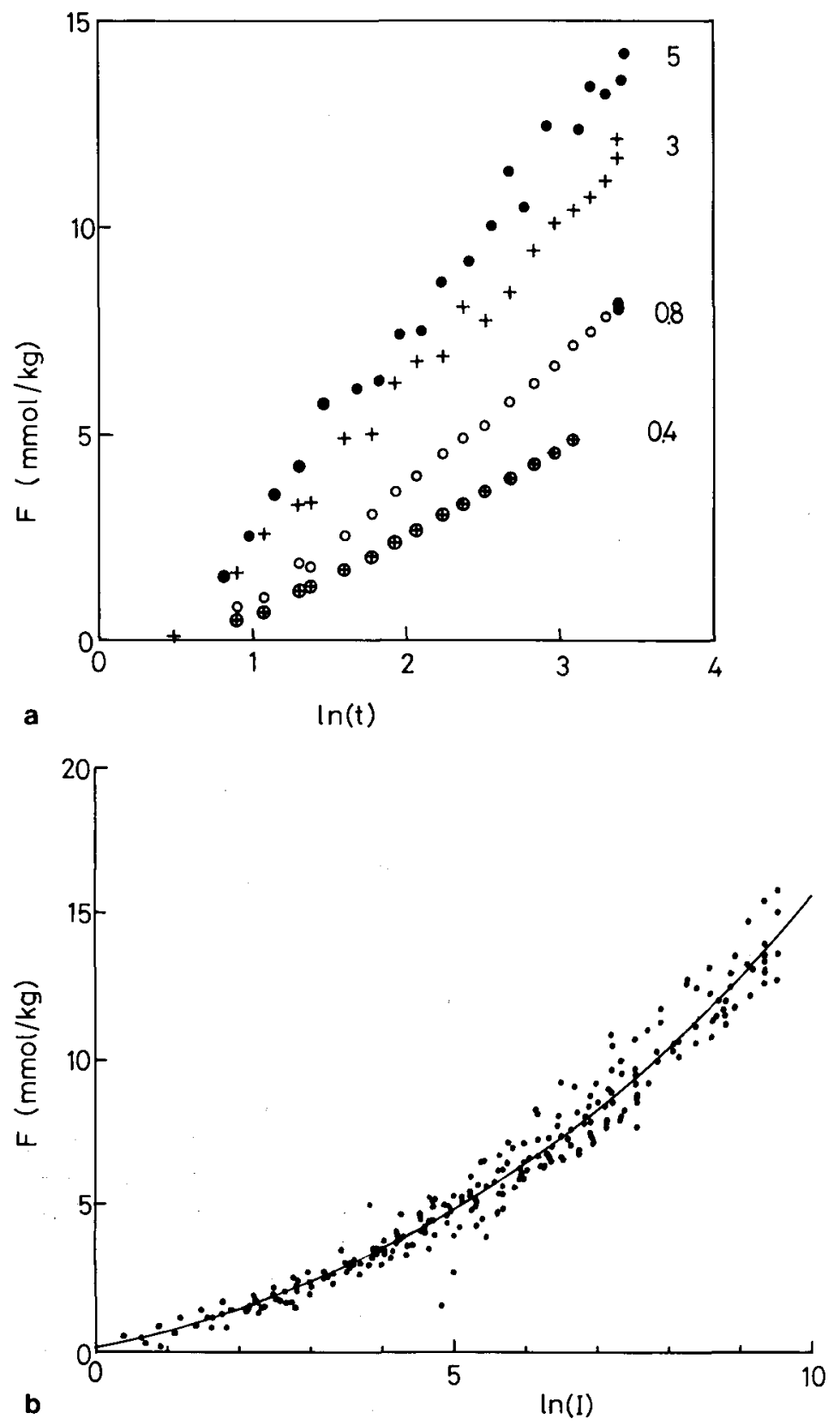

Fig. 2. Amount of phosphate that has reacted with soil as a function of the natural logarithm of time (2a). The concentrations are indicated in $\mathrm{mol} \mathrm{m}^{-3}$ in the figure. Same amounts as a function of the natural logarithm of exposure $(\ln I)$ for phosphate concentrations in the range $0.05-6 \mathrm{~mol} \mathrm{~m}^{-3}(2 \mathrm{~b})$. Solid curve is the best fitting third-degree polynomial, $c_{e}=0.1 \mathrm{~mol} \mathrm{~m}^{-3}$. Time in min. Points with $\ln (I)$ values smaller than 0 were omitted. 


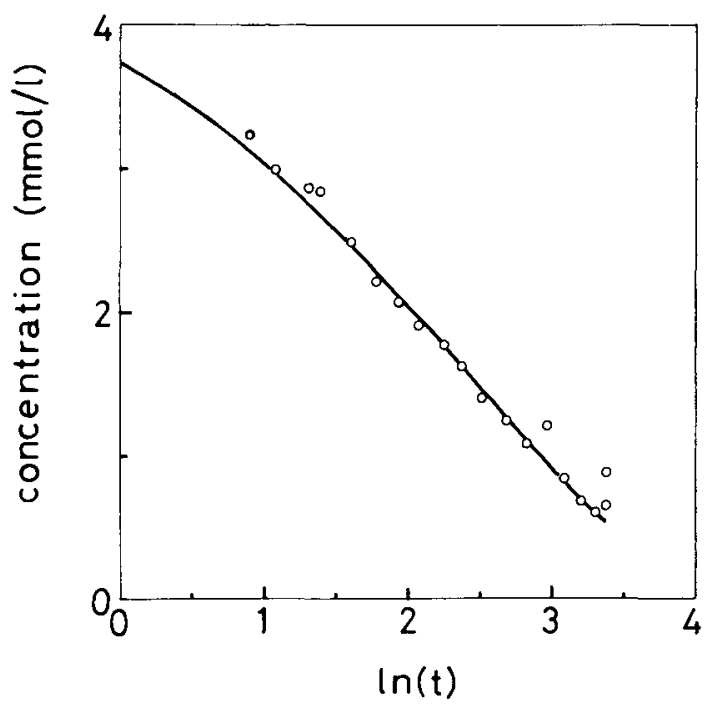

Fig. 3. Phosphate concentration in solution $(c)$ as a function of time $(t)$. Experimental points and curve as calculated with the empirical polynomial of Fig. 2.

ecological functioning of the soil. Infiltration of atmospheric pollutants causes a complex chain of exchange and mineral weathering reactions in the soil. On the long term, mineral weathering controls the soil $\mathrm{pH}$ and nutrient status of unfertilized soils. Mineral weathering is still a poorly understood process (Helgeson et al., 1984). Most research concerning mineral weathering dealt with pure minerals such as feldspar (Helgeson et al., 1984; Chou \& Wollast, 1984). Mainly two mechanisms of weathering have been proposed. The first type conforms to the USC model, either with a product layer that continually increases in thickness (Wollast, 1967; Helgeson, 1971, 1972) or with a constant thickness caused by dissolution of the product layer at the particle surface (Correns, 1963; Paces, 1973). In an alternative description, feldspar dissolution is assumed to be controlled by a surface reaction and incongruent dissolution is attributed to precipitation of aluminum hydroxides or aluminum silicates (Busenberg, 1978; Holdren \& Adams, 1982; Helgeson et al., 1984). In models of the second type, the commonly observed parabolic-type kinetics are attributed to e.g. exchange reactions, etch pitch formation, mineral impurity, or particle size heterogeneity. Little consensus exists on the microscopic mechanisms that cause the overall kinetics of feldspar weathering, so a mechanistic description of the rate of proton consumption in the more complex soil system is still remote. Nevertheless, a description of the proton consumption rate is desirable to estimate long-term effects of atmospheric pollutant deposition. Currently, predictions of such long-term effects are usually based on the extrapolation of empirical rate equations (Cosby, 1986).

To investigate whether the time scaling proposed here may yield an additional in- 


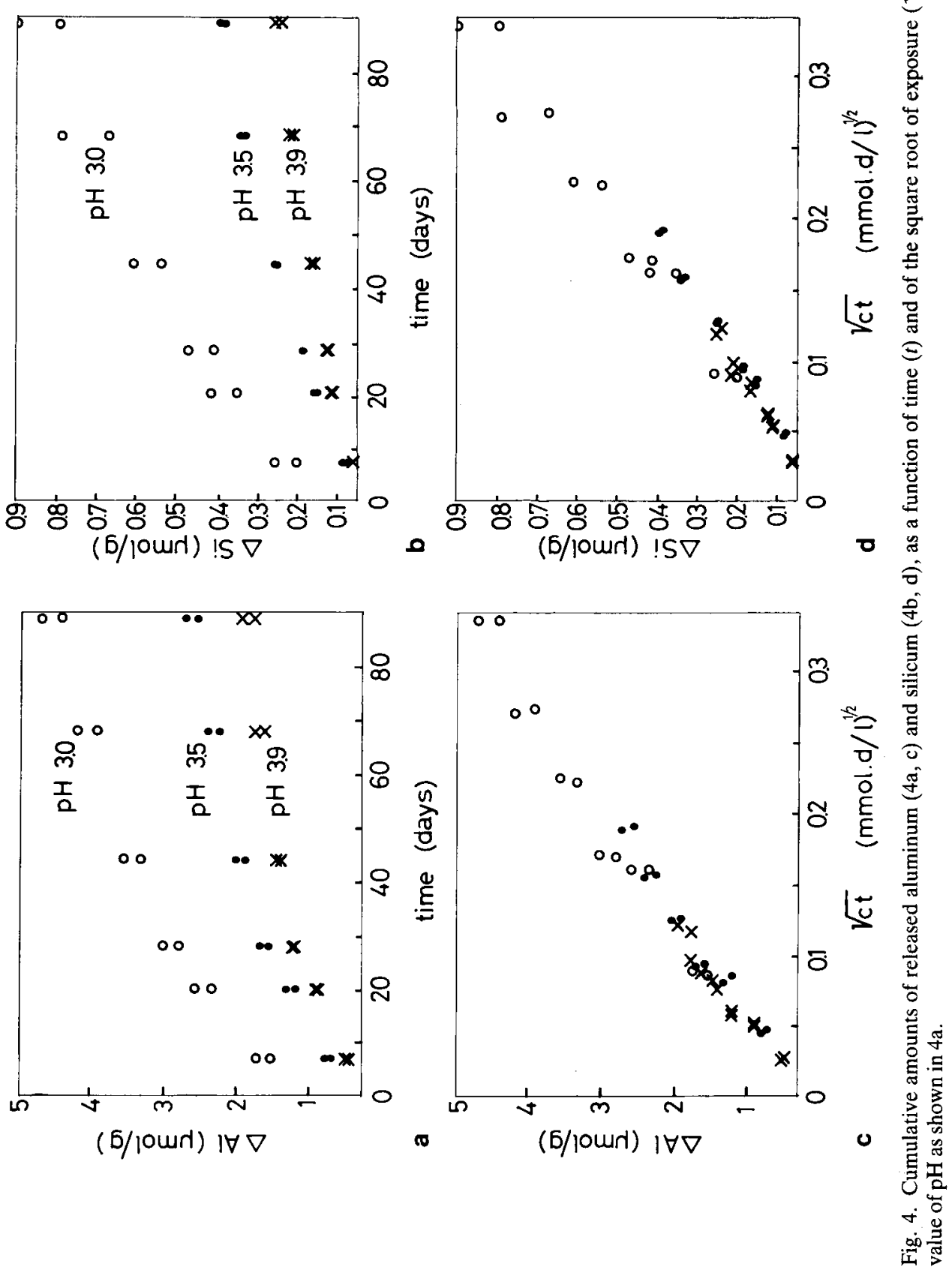


strument for predictions of soil acidification, soil columns were leached with solutions with a particular constant, low $\mathrm{pH}$ at relatively high flow rates, varied to maintain a constant $\mathrm{pH}$ in the soil columns (van Grinsven, 1988). This was done for sandy subsoil samples (70-80 $\mathrm{cm}$ depth) (van Grinsven, 1988). In this soil, proton consumption is predominantly caused by the reaction with various silicates and aluminum (hydr)oxides present, resulting in the release of aluminum, calcium and silicium and small amounts of potassium, magnesium, and sodium. When the reaction rates had slowed down considerably after a number of days, the experiments were continued in batch for a period of 90 days. The reaction vessels were not shaken and the $\mathrm{pH}$ was kept constant by regularly replacing the supernatant solution (every one to three weeks). In the batch experiments, $\mathrm{Al}$ and $\mathrm{Si}$ were the main compounds that were released. The column and batch experiments were done at constant $\mathrm{pH}$ values of $3,3.5$ and 3.9. The widely divergent curves showing the amount of a compound released as a function of time for the different solution $\mathrm{pH}$ values, scale very well for the main compounds in both the batch experiments (Figure 4) and the column experiments (not shown). The reproducibility appears to be good too as is seen from the duplicated experimental data in Figure 4. With these results the question to which mechanism or combination of mechanisms causes the observed weathering rates is not answered. In fact, regardless of the mechanisms of the feldspar weathering discussed in the literature the experimental results may be scaled with the exposure variable, $I$, provided the reaction rate is assumed to be first-order with respect to the proton concentration, and rate controlling.

For the assessment of long-term effects of soil acidification, the scaling procedure may yield more reliable results than purely empirical descriptions (Cosby, 1986).

\section{Conclusions}

In this paper we discussed the scaling of time in the case of reactions whose rates are controlled by reactant diffusion, a first-order chemical reaction or a combination of these processes. The scaling procedure proposed that has at most one adjustable parameter $\left(c_{e}\right)$ is derived from the unreacted shrinking core (USC) model. A unique relation is found between the amount of reactant or solid phase that has reacted and an exposure variable, which is a function of concentration and time. Moreover, the scaling rule is also valid for other diffusion-controlled processes, e.g., when abrasion of the product layer occurs, in case of irregular solid phase geometry, when various particle sizes occur or when the composition of the solid phase and the reaction stoichiometry vary as in soil. These and other complications do not affect the possibility to apply our scaling procedure. The scaling procedure may be useful in a semi-empirical basis for interpolation and extrapolation in time for complex real-world reaction systems that are not (yet) amenable to a mechanistic description. Once the relationship between conversion and exposure had been established experimentally, the conversion as a function of time and concentration may be calculated. Hence, the scaling rule may be a useful tool for the simulation of certain real-world problems, either in scientific research or for management purposes. 


\section{Acknowledgement}

We thank Dr N. van Breemen (Department of Soil Science and Geology, Wageningen Agricultural University) for his constructive criticism of the manuscript.

\section{References}

Barrow, N. J., 1983. A mechanistic model for describing the sorption of phosphate by soil. Journal of Soil Science 34: 733-750.

Bartlett, R. W., 1971. Conversion and extraction efficiencies for ground particles in heterogeneous process reactors. Metallurgical Transactions 2: 2999-3006.

Bartlett, R. W., 1973. A combined pore diffusion and chalcopyrite dissolution kinetics model for in site leaching of a fragmented copper porphyry. In: Proceedings International Symposium Hydrometallurgy, American Institute Mining Engineering, New York, p. 331-374.

Beek, J., 1979. Phosphate retention by soil in relations to waste disposal. Doctoral Thesis, Wageningen Agricultural University, $162 \mathrm{pp}$.

Bird, R. B., W. E. Stewart \& E. N. Lightfoot, 1960. Transport phenomena, John Wiley and Sons Inc., New York, $780 \mathrm{pp}$.

Bischoff, K. B., 1963. Accuracy of the pseudo steady state approximation for moving boundary diffusion problems. Chemical Engineering Science 18: 711-713.

Braun, R. L., A. E. Lewis \& M. E. Wadsworth, 1964. In-place leaching of primary sulfide ores: Laboratory leaching data and kinetics model. Metallurgical Transactions 5: 1717-1726.

Brunekreeff, B., 1985. The relationship between environmental lead and blood lead in children: a study in environmental epidemology. Doctoral Thesis, Wageningen Agricultural University.

Busenberg, E., 1978. The products of the interaction of feldspars with aqueous solutions at $25^{\circ} \mathrm{C}$. Geochimica et Cosmochimica Acta 42: 1679-1686.

Chou, L. \& R. Wollast, 1984. Study of the weathering of albite at room temperature and pressure with a fluidized bed reactor. Geochimica et Cosmochimica Acta 48: 2205-2217.

Correns, C. W., 1963. Experiments on the decomposition of silicates and discussion of chemical weathering. Clays and Clay Mineralogy 10: 443-459.

Cosby, B. J., et al., 1986. Estimating catchment water quality response to acid deposition using mathematical models of soil ion exchange processes. Geoderma 38: 77-95.

Davis, G. B. \& J. M. Hill, 1982. A moving boundary problem for the sphere. I.M.A. Journal Applied Mathematics 22: 99-111.

Enfield, C. G., T. Phan, D. M. Wolters \& R. Ellis Jr, 1981. Kinetic model for phosphate transport and transformation in calcareous soils, I and II. Soil Science Society American Journal 45: 1059-1064; 1064-1070.

Grinsven, J. J. M. van, 1988. Impact of acid atmospheric deposition on soils. Quantification of chemical and hydrologic processes. Doctoral Thesis, Wageningen Agricultural University, $215 \mathrm{pp}$.

Helgeson, H. C., 1971. Kinetics of mass transfer among silicates and aqueous solutions. Geochimica et Cosmochimica Acta 35: 421-469.

Helgeson, H. C., 1972. Kinetics of mass transfer among silicates and aqueous solutions: correction and clarification. Geochimica et Cosmochimica Acta 36: 1067-1070.

Helgeson, H. C., W. M. Murphy, P. Aagaard, 1984. Thermodynamic and kinetic constraints on reaction rate among minerals and aqueous solutions. II. Rate constants, effective surface area and the hydrolysis of feldspar. Geochimica et Cosmochimica Acta 48: 2405-2432.

Hiskey, J. B. \& M. E. Wadsworth, 1974. Galvanic conversion of chalcopyrite. In: F. F. Aplan, W. A. McKinney \& A. D. Pernichele (Eds), Solution Mining Symposium, American Institute Mining Engineering, New York: p. 422-445.

Holdren, G. R., Jr \& J. E. Adams, 1982. Parabolic dissolutions kinetics of silicate minerals: an artifact of non-equilibrium precipitation processes? Geology 10: 186-190.

Levenspiel O., 1972. Chemical reaction engineering. Chapter 12, 2nd ed. John Wiley and Sons Inc., Canada. 
$\mathrm{Lu}, \mathrm{W} . \mathrm{K}$, 1963. The general rate equation for gas-solid reactions in metallurgical process. Transactions Metallurgical Society American Institute Mining Engineering 227: 203-206.

Lu, W. K. \& G. Bitsianes, 1966. The general rate equation for gas-solid reactions in metallurgical processes. II. With restrictions of reversibility of chemical reaction and gaseous equimolal counter diffusion. Transactions Metallurgical Society American Institute Mining Engineering 236: 531-535.

Ockendon, J. R. \& W. R. Hodgkins (Eds), 1965. Moving boundary problems in heat flow and diffusion. Clarendon Press, Oxford.

Paces, T., 1973. Steady state kinetics and equilibrium between groundwater and granitic rock. Geochimica et Cosmochimica Acta 37: 2641-2663.

Rahman, Z. B. A., 1982. The fate of phosphate fertilizer in Malaysian soils and its effect on plant uptake. Doctoral Thesis, University of Gent, Belgium.

Riemsdijk, W. H. van \& A. M. A. van der Linden, 1984. Phosphate sorption by soil. II. Sorption measurement technique. Soil Science Society American Journal 48: 541-544.

Shen, J. \& J. M. Smith, 1965. Diffusional effects in gas-solid reactions. Industrial Engineering Chemistry Fundamentals 4: 293-301.

Skopp, J., 1986. Analysis of time-dependent chemical processes in soils. Journal Environmental Quality 15: 205-213.

Szekely, J. \& M. Propster, 1975. A structural model for gas solid reactions with a moving boundary. VI. The effect of grain size distribution on the conversion of porous solids. Chemical Engineering Science 30: 1049-1055.

Wen, C. Y., 1968. Non-catalytic heterogeneous solid fluid reaction models. Industrial Engineering Chemistry 60: 34-54.

Wollast, R., 1967. Kinetics of the alteration of K-feldspar in buffered solutions at low temperature. Geochimica et Cosmochimica Acta 31: 635-648.

Yagi, S. \& D. Kunii, 1984. Combustion of carbon particles in flames and fluidized beds. Symposium on Combustion Proceedings, p. 231-244. Pittsburgh.

Zee, S. E. A. T. M. van der, 1988. Transport of reactive contaminants in heterogenous soil systems. Doctoral Thesis, Wageningen Agricultural University, $283 \mathrm{pp}$.

Zee, S. E. A. T. M. van der \& W. H. van Riemsdijk, 1986. The reaction of phosphate described with adsorption in combination with the unreacted shrinking cure model. Conference 'Interactions at the soilcolloid soil-solution interface', Ghent, Belgium.

Zee, S. E. A. T. M. van der \& W. H. van Riemsdijk, 1988. Model for long-term phosphate reaction kinetics in soil. Journal Environmental Quality 17: 35-41. 